Check for updates

The BMJ

Cite this as: $B M J 2022 ; 376: 0428$ http://dx.doi.org/10.1136/bmj.0428 Published: 18 February 2022

\title{
Covid-19: Antibodies after AstraZeneca and Pfizer vaccines decrease with age and are higher in women, data show
}

Gareth lacobucci

SARS-CoV-2 antibody levels after receiving the AstraZeneca or Pfizer-BioNTech vaccine decrease with age and are higher in females and people with prior infection, show data from the Real-time Assessment of Community Transmission (React-2) study. ${ }^{1}$

The study, led by Imperial College London, analysed self-reported results from Fortress lateral flow tests to detect antibodies in a drop of blood from a finger prick. Data were collected from 212102 adults from January to May 2021, of whom 71923 (33.9\%) had received at least one dose of Pfizer-BioNTech and 139 067 (65.6\%) at least one dose of AstraZeneca.

Results published in Nature Communications showed that, after either of the vaccines, antibody positivity peaked four to five weeks after the first dose and then declined until after second doses were given. ${ }^{1}$ "For both vaccines, there was a clear increase in the proportion of individuals testing positive after second doses," the researchers said.

Of 68 o6o adults who had received their second vaccine dose at least 21 days earlier, nearly $100 \%$ of respondents had antibodies to the virus after a second dose of Pfizer. The figure dropped significantly in people who had AstraZeneca, particularly in the oldest age groups $(72.7 \%$ (95\% confidence interval $70.9 \%$ to $74.4 \%$ ) at age 75 and above). Overall, antibody positivity was higher in those who had received the Pfizer vaccine rather than AstraZeneca (odds ratio 3.67 (3.49 to 3.85)).

Data on both vaccines showed that antibody positivity was lower in older people, with an odds ratio of 0.30 (0.24 to 0.37) in those aged over 75 versus those aged 35-44; higher in women than men (1.37 (1.30 to 1.43)); and higher in people with prior infection (2.39 (2.18 to 2.63$)$ ).

After two vaccine doses, antibody positivity was substantially lower (0.16 (0.12 to 0.22)) in people who reported being an organ transplant recipient or having a weakened immune system from illness or treatment. Positivity was also lower in people with diabetes, stroke, kidney, liver, lung or neurological disease, cancer, and depression.

\section{Key groups}

The researchers said, "These population data confirm the importance of second vaccine doses and provide strong evidence for the role of individual factors (particularly age, sex, prior infection, adiposity and comorbidities) and vaccine type in determining antibody response, particularly after first doses.

"Whilst simplicity is key to successful vaccine rollout, this information identifies key groups that may benefit from additional vaccine doses when available."

But they added that antibody positivity was "only one measure of a multifaceted immune response," noting that $\mathrm{T}$ cell response persists for at least six to eight months and that $B$ cell mediated immunity can be sustained at least 12 months after initial infection.

They concluded, "It is possible many individuals will have sufficiently preserved immunity after two vaccine doses to prevent severe disease, but further follow-up is required to determine the longevity of protection."

1 Ward H, Whitaker M, Flower B, et al. Population antibody responses following COVID-19 vaccination in 212,102 individuals. Nature Comms 2022;13:907. https://www.nature.com/articles/s41467-022-28527-x

This article is made freely available for personal use in accordance with BMJ's website terms and conditions for the duration of the covid-19 pandemic or until otherwise determined by BMJ. You may download and print the article for any lawful, non-commercial purpose (including text and data mining) provided that all copyright notices and trade marks are retained. 